\title{
Stabilisation of non-linear networked control systems closed over a lossy WirelessHART network
}

\author{
Alejandro I. Maass and Dragan Nešić
}

\begin{abstract}
This paper studies the stabilisation of non-linear networked control systems (NCSs) where the information between plant and controller is sent over a lossy wireless multihop network under a carrier-sense multiple access with collision avoidance (CSMA-CA) scheme. We present a hybrid model for the overall NCS that captures time-varying transmission instants, both inter- and at-transmission behaviour, packet dropouts, field device dynamics, and CSMA-CA scheduling. We then use this model to provide sufficient conditions in terms of the intensity of transmission that ensure closed-loop $\mathcal{L}_{p}$ stability-in-expectation. In doing so, we exploit the mathematical structure of our NCS model to improve previous results in the literature.
\end{abstract}

Index Terms-Networked control systems, stability of nonlinear systems, control over communications

\section{INTRODUCTION}

W IRELESS networks naturally introduce stochastic behaviour into the control loop that may deteriorate its performance and stability [1]. In this paper, we focus on networked control systems (NCSs) implemented over wireless multi-hop networks, which are increasingly used in industry control. Particularly, we study WirelessHART (WH), the first open wireless communication standard for measurement and control in the process industries. According to many international companies, it serves as a compelling alternative for industrial control, as it improves operations, increases productivity, and saves money [2]. NCSs implemented over multi-hop networks have received a lot of theoretical attention in the last decade, see e.g. [3], [4] and the references therein. In WH, the two most common stochastic phenomena are packet losses and carrier-sense multiple access with collision avoidance (CSMACA) schemes. Packet losses are particularly interesting in the context of wireless networks, and they are typically caused by network congestion, faulty hardware, weak radio signals, or even intentionally dropped by routing routines. CSMA-CA lies in WH's media access control (MAC) layer, which is the lower layer within the data link layer that controls when and how to send and receive packets. WH may also use timedivision multiple access (TDMA), which was studied in our previous work [5], [6]. That is, each device is scheduled to transmit in a specific timeslot deterministically. In this work we restrict our attention to CSMA-CA schemes, in which more than one device may try to transmit at the same time on the same channel. Only one field device will gain access to the

This work was supported by the Australian Research Council under the Discovery Project DP170104099.

A.I. Maass and D. Nešić are with the Department of Electrical and Electronic Engineering, The University of Melbourne, Parkville, 3010, Victoria, Australia. amaassestudent.unimelb.edu.au, dnesic@unimelb.edu.au timeslot whenever the channel is determined to be clear. The rest will retry to transmit as soon as the channel is clear again.

Relevant results in the literature that consider wireless NCSs (WNCSs) under packet dropouts and CSMA/CA include [3], [4], [7]-[12]. The works [3], [4], [7]-[11] consider discretetime linear plants and controllers, and their network models use equidistant transmission instants, which can be considered strong assumptions when dealing with wireless systems. Nonlinear WNCSs under dropouts and CSMA/CA are studied in [12], however, their results are not tailored to multi-hop networks and are then often conservative when applied to such networks as we illustrate in this paper.

In this paper, we present a more general modelling framework than [3], [4], [7]-[11] that includes non-linear plant and controller, time-varying transmission instants, both interand at-transmission behaviour, packet dropouts, field device dynamics, and CSMA-CA scheduling. A key feature of our WNCS model is that the multi-hop nature of the network is reflected as a specific mathematical structure in the networkinduced error dynamics, as opposed to [12]. We then follow the emulation approach [13], [14] to obtain sufficient conditions for closed-loop $\mathcal{L}_{p}$ stability-in-expectation of our WNCS model. In particular, we adopt appropriate requirements on the scheduling protocol such that the WNCS is $\mathcal{L}_{p}$ stable-inexpectation for sufficiently high transmission rates. We adopt a property that is the stochastic analogue to the persistently exciting (PE) property introduced in [15], i.e. we consider a property that is such that every field device almost surely transmits within a finite number of transmissions $T$. The (now random) variable $T$ is closely related to the cover time in an undirected graph [16], and the running time of the Coupon Collector's problem [17]. Although cover times and transmission instants are now random, and hence not uniform, if the network-free system is $\mathcal{L}_{p}$ stable, the WNCS remains so with any admissible protocol whenever attempted transmissions occur "fast enough". With "fast enough" we mean that there exists a choice of intensity $\boldsymbol{\omega}$ of the transmission process parametrised by properties of the protocol and the WNCS dynamics, such that the WNCS is $\mathcal{L}_{p}$ stable-in-expectation from disturbance to NCS state with a finite expected gain. Furthermore, in our analysis we exploit the mathematical structure of our model and thus provide less conservative intensity bounds than the ones that can be obtained with the generic framework [12]. Although our results are presented for a particular wireless multi-hop network (i.e. WH), these can be applied to other multi-hop networks with minor modifications as we detail later. 


\section{PRELIMINARIES}

\section{A. Notation}

Denote by $\mathbb{R}$ the set of real numbers, $\mathbb{R}^{n}$ the set of all real vectors with $n$ components, $\mathbb{Z}_{\geq 0} \doteq\{0,1,2, \ldots\}$ and $\mathbb{N} \doteq\{1,2, \ldots\}$. For vector arguments, $|\cdot|$ denotes the Euclidean norm. We use $(x, y) \doteq\left[x^{T} y^{T}\right]^{T} \in \mathbb{R}^{n+m}$, for all $x \in \mathbb{R}^{n}$ and $y \in \mathbb{R}^{m}$. Given $t \in \mathbb{R}$ and a piecewise continuous function $f: \mathbb{R} \rightarrow \mathbb{R}^{n}$, we use the notation $f\left(t^{+}\right) \doteq \lim _{s \rightarrow t, s>t} f(s) . I_{n}$ stands for the $n \times n$ identity matrix. We use $\mathbf{I}_{n}^{N} \doteq\left[\begin{array}{lll}I_{n} & \cdots & I_{n}\end{array}\right]^{N}$ to denote the matrix $\left[\begin{array}{lll}I_{n} & \cdots & I_{n}\end{array}\right] \in \mathbb{R}^{n \times N n}$. We define $\bar{x} \doteq\left(\left|x_{1}\right|, \ldots,\left|x_{n}\right|\right)^{T}$ for all $x \in \mathbb{R}^{n}$. For a function $f: \mathbb{R} \rightarrow \mathbb{R}^{n}$, we define $\bar{f}: t \rightarrow \overline{f(t)}$. Let $f: \mathbb{R} \rightarrow \mathbb{R}^{n}$ be a (Lebesgue) measurable function and define $\|f\|_{\mathcal{L}_{p}} \doteq\left(\int_{\mathbb{R}}|f(s)|^{p} d s\right)^{1 / p}$, for $p \in \mathbb{N}$, and define $\|f\|_{\mathcal{L}_{\infty}} \doteq \operatorname{ess~sup}_{t \in \mathbb{R}}\|f(t)\|$, and $\|f\|_{\mathcal{L}_{\infty}[a, b]} \doteq$ $\operatorname{ess~sup}_{t \in[a, b]}\|f(t)\|$. We say that $f \in \mathcal{L}_{p}$ for $p \in[1, \infty]$ whenever $\|f\|_{\mathcal{L}_{p}}<\infty$. Let $f: \mathbb{R} \rightarrow \mathbb{R}^{n}$ and let $[a, b] \subset \mathbb{R}$, we use the notation $\|f\|_{\mathcal{L}_{p}[a, b]} \doteq\left(\int_{[a, b]}\|f(s)\|^{p} d s\right)^{1 / p}$. Let $\mathcal{A}_{\geq 0}^{n}$ denote the set of all $n \times n$ matrices with nonnegative entries, and let $\mathbb{R}_{\geq 0}^{n}$ denote the non-negative orthant of $\mathbb{R}^{n}$. Let $x=\left(x_{1}, \ldots, x_{n}\right), y=\left(y_{1}, \ldots, y_{n}\right) \in \mathbb{R}^{n}$. A partial order $\preceq$ is given by $x \preceq y \Longleftrightarrow x_{i} \leq y_{i}$, for all $i \in\{1, \ldots, n\}$. A partial order on elements of $\mathcal{A}_{\geq 0}^{n}$ is defined as $A \preceq B \Longleftrightarrow B-A \in \mathcal{A}_{\geq 0}^{n} . \mathbf{P}\{\cdot\}$ and $\mathbf{E}\{\cdot\}$ stand for the probability and expected value operator, respectively. Given a continuous random variable $X$ the associated moment generating function (mgf) is given by $\mathbf{M}_{X}(t) \doteq \mathbf{E}\{\exp (t X)\}$. For a discrete random variable $Y$ taking values in $\mathbb{Z}_{\geq 0}$, we define the probability generating function (pgf) as $\mathbf{G}_{Y}(s) \doteq \mathbf{E}\left\{s^{Y}\right\}$.

\section{B. Stability notion}

We will consider systems with stochastic impulses

$$
\begin{aligned}
\dot{z} & =f(t, z, w), t \in\left[t_{i}, t_{i+1}\right], \\
z\left(t_{i}^{+}\right) & =\mathcal{H}(i) z\left(t_{i}\right), \\
y & =G(t, z),
\end{aligned}
$$

where $z \in \mathbb{R}^{n_{z}}$ is the state, $w \in \mathbb{R}^{n_{w}}$ is an exogenous perturbation, $y \in \mathbb{R}^{n_{y}}$ is a prescribed output, $n_{z}, n_{w}, n_{y} \in \mathbb{N}$, $\{\mathcal{H}(i)\}_{i \in \mathbb{N}}$ is a sequence of random matrices, and $\left\{t_{i}\right\}_{i=0}^{\infty}$ is a sequence of random increasing time instants. Suppose (1) is initialised at $\left(t_{0}, z_{0}\right)$ with input $w$. We consider the following stability notion for systems like (1).

Definition 1: Let $p \in[1, \infty]$ and $\gamma \geq 0$ be given. We say that (1) is $\mathcal{L}_{p}$ stable-in-expectation from $w$ to $y$ with expected gain $\gamma$ if there exists $K \geq 0$ such that $\mathbf{E}\left\{\|y\|_{\mathcal{L}_{p}\left[t_{o}, t\right]}\right\} \leq K\left|z_{0}\right|+$ $\gamma \mathbf{E}\left\{\|w\|_{\mathcal{L}_{p}\left[t_{0}, t\right]}\right\}$, for all $t \geq t_{0}$.

\section{Problem Statement}

In this paper, we follow the emulation approach in e.g. [13], [14], which we explain below. Consider the non-linear plant $\dot{x}_{p}=f_{p}\left(x_{p}, u, w\right), y=g_{p}\left(x_{p}\right)$, where $x_{p} \in \mathbb{R}^{n_{p}}$ is the state, $u \in \mathbb{R}^{n_{u}}$ is the control signal, $y \in \mathbb{R}^{n_{y}}$ is the plant output, $w \in$ $\mathbb{R}^{n_{w}}$ is an exogenous perturbation which is assumed to belong to $\mathcal{L}_{p}$, and $n_{p}, n_{u}, n_{y}, n_{w} \in \mathbb{N}$. The first step of emulation is to assume a controller $\dot{x}_{c}=f_{c}\left(x_{c}, y, w\right), u=g_{c}\left(x_{c}\right)$ has

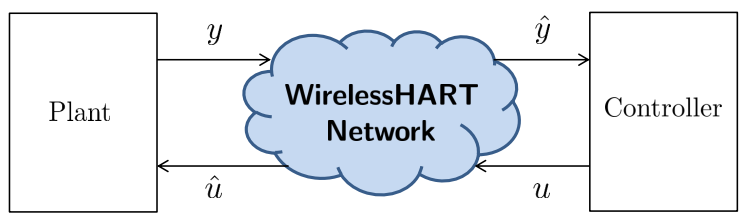

Fig. 1. Block diagram of our networked control architecture.

been designed to stabilise the above plant, where $x_{c} \in \mathbb{R}^{n_{c}}$ is the state of the controller, $y \in \mathbb{R}^{n_{y}}$ is the plant output, $u \in \mathbb{R}^{n_{u}}$ is the control signal, and $n_{c} \in \mathbb{N}$. The functions $f_{p}, f_{c}$ are assumed to be continuous and $g_{p}, g_{c}$ are assumed to be continuously differentiable.

Then, as the second step of emulation, we implement such controller over a WH network as per Fig. 1. We note that now the controller has only access to the networked version of $y$, denoted by $\hat{y}$, and the plant now receives the networkedversion of the control signal $u$, denoted by $\hat{u}$. Particularly, we have that

$$
\begin{aligned}
& \dot{x}_{p}=f_{p}\left(x_{p}, \hat{u}, w\right), \quad y=g_{p}\left(x_{p}\right), \\
& \dot{x}_{c}=f_{c}\left(x_{c}, \hat{y}, w\right), \quad u=g_{c}\left(x_{c}\right) .
\end{aligned}
$$

After the controller is implemented over the network, our aim is to provide conditions on the controller and the network, under which the stability property obtained by the controller without the network, is preserved when such controller is implemented over the network. Particularly, we are interested in conditions under which the closed-loop in Fig. 1 is $\mathcal{L}_{p}$ stable-in-expectation as per Definition 1.

\section{NETWORK MODELLING}

\section{A. Specifications}

WH is a multi-hop network that consists of an interconnection of field devices, which include sensors, actuators, and routers. It is based on the IEEE 802.15.4-2006 physical layer and operates in the $2.4 \mathrm{GHz}$ ISM radio band over 15 frequency division multiplexed channels. Each frequency channel is subdivided into timeslots in which only one field device is allowed to transmit. We restrict our attention to $\mathrm{WH}$ networks that use CSMA-CA schemes for scheduling, which are naturally stochastic as we explain further below.

For each $i$-th timeslot, we denote the corresponding transmission instant by $t_{i}$. In wireless networks, transmission instants are naturally time-varying and random, we thus adopt the following assumption.

Assumption 1: Consider a Poison point process $N(t)$ with intensity $\boldsymbol{\omega}$ that satisfies $N(t)=0$ for $t \in\left[0, t_{0}\right)$ and $N(t)=i$ for $t \in\left[t_{i-1}, t_{i}\right)$. Consequently, the sequence of transmission instants $\left\{t_{i}\right\}_{i \in \mathbb{N}}$ is defined inductively by: $t_{0}=\tau_{0}$ with $\tau_{0} \sim$ $\operatorname{Exp}(\boldsymbol{\omega})$, and for each $i>0, t_{i}=t_{i-1}+\tau_{i}$, with $\tau_{i} \sim \operatorname{Exp}(\boldsymbol{\omega})$, where the sequence $\left\{\tau_{i}\right\}_{i \in \mathbb{N}}$ is i.i.d.

The exponential distribution that governs $\tau_{i}$ is the probability distribution that describes the time between events (transmissions) in the Poisson point process $N(t)$. 


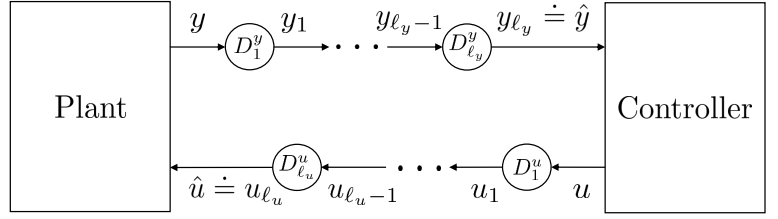

Fig. 2. NCS implemented over a WH network with $\ell_{y}$ field devices in the plant-to-controller link and $\ell_{u}$ field devices in the controller-to-plant link.

\section{B. Field devices}

Consider Fig. 2, where the network is composed of $\ell_{y} \in$ $\mathbb{Z}_{\geq 0}$ field devices interconnected in the plant-to-controller path (y-path), and $\ell_{u} \in \mathbb{Z}_{\geq 0}$ in the controller-to-plant path (u-path). We label the field devices as $D_{\alpha}^{y}$ and $D_{\beta}^{u}$, where $\alpha=1, \ldots, \ell_{y}$ and $\beta=1, \ldots, \ell_{u}$, see Fig. 2. According to WH specifications, each field device is simplex (i.e. it cannot receive and transmit at the same time) and acts as a router for data from/to neighbouring field devices. Consequently, we model field devices as buffers, for which we introduce buffer state variables, denoted by $b_{\alpha}^{y}$ and $b_{\beta}^{u}$ for field devices in the $y$-path and $u$-path, respectively. In the sequel, we explain the reception and transmission behaviour of field devices, and we present the equations associated with this process.

Reception: Suppose a field device $D_{\alpha}^{y}$ receives a packet at time instant $t_{i}$. Then, $D_{\alpha}^{y}$ updates the content of its buffer via its input. During this process, the output of $D_{\alpha}^{y}$ remains unchanged. We write this as follows,

$$
\begin{aligned}
\dot{y}_{\alpha}(t) & =0, \quad \dot{b}_{\alpha}^{y}(t)=0, \quad t \in\left[t_{i}, t_{i+1}\right], \\
b_{\alpha}^{y}\left(t_{i}^{+}\right) & =y_{\alpha-1}\left(t_{i}^{+}\right), \quad y_{\alpha}\left(t_{i}^{+}\right)=y_{\alpha}\left(t_{i}\right),
\end{aligned}
$$

for all $\alpha=1, \ldots, \ell_{y}$. Note that $y_{0} \equiv y$ for $\alpha=1$, i.e. device one samples the value of the plant output.

Transmission: Suppose a field device $D_{\alpha}^{y}$ is scheduled to transmit at time instant $t_{i}$. Here, $D_{\alpha}^{y}$ sends the content of its buffer through its output, and keeps it until a new packet is received. This can be written as follows,

$$
\begin{aligned}
\dot{y}_{\alpha}(t) & =0, \quad \dot{b}_{\alpha}^{y}(t)=0, \quad t \in\left[t_{i}, t_{i+1}\right], \\
y_{\alpha}\left(t_{i}^{+}\right) & =b_{\alpha}^{y}\left(t_{i}\right), \quad b_{\alpha}^{y}\left(t_{i}^{+}\right)=b_{\alpha}^{y}\left(t_{i}\right),
\end{aligned}
$$

for all $\alpha=1, \ldots, \ell_{y}$.

Motivated by (3)-(4), and to simplify analysis, we introduce the network-induced error, which we denote by $\zeta \in \mathbb{R}^{n_{\zeta}}$, with $n_{\zeta} \doteq n_{\zeta^{y}}+n_{\zeta^{u}}, n_{\zeta^{y}} \doteq 2 \ell_{y} n_{y}$, and $n_{\zeta^{u}} \doteq 2 \ell_{u} n_{u}$. We define it as $\zeta \doteq\left(\zeta^{y}, \zeta^{u}\right)$, where $\zeta^{y} \in \mathbb{R}^{n_{\zeta^{y}}}$ and $\zeta^{u} \in \mathbb{R}^{n_{\zeta^{u}}}$ are the corresponding errors of the $y$-path and $u$-path, respectively, and are given by

$$
\begin{gathered}
\zeta^{y} \doteq\left(b_{1}^{y}-y, b_{2}^{y}-y_{1}, \ldots, b_{\ell_{y}}^{y}-y_{\ell_{y}-1},\right. \\
\left.y_{1}-b_{1}^{y}, y_{2}-b_{2}^{y}, \ldots, y_{\ell_{y}}-b_{\ell_{y}}^{y}\right), \\
\zeta^{u} \doteq\left(b_{1}^{u}-u, b_{2}^{u}-u_{1}, \ldots, b_{\ell_{u}}^{u}-u_{\ell_{u}-1},\right. \\
\left.u_{1}-b_{1}^{u}, u_{2}-b_{2}^{u}, \ldots, u_{\ell_{u}}-b_{\ell_{u}}^{u}\right) .
\end{gathered}
$$

The first $\ell_{\star}$ components of $\zeta^{\star}, \star \in\{y, u\}$, are related to the buffer update during reception, and the remaining $\ell_{\star}$ components of $\zeta^{\star}$ are related to the transmission of such buffer value through their output. In particular, we zero components of these errors whenever a transmission occurs.

\section{CSMA-CA scheduling}

In CSMA-CA schemes, several devices try to gain access to the timeslot, but only one will succeed when the channel is determined to be clear. The intuition behind the modelling we present below is that at each transmission instant and randomly, one of the contending field devices $D_{1}^{y}, \ldots, D_{\ell_{y}}^{y}, D_{1}^{u}, \ldots, D_{\ell_{u}}^{u}$ will access the timeslot and thus have its corresponding error components set to zero.

We consider the following equation to model transmissions

$$
\zeta\left(t_{i}^{+}\right)=\mathcal{H}(i) \zeta\left(t_{i}\right),
$$

where $\mathcal{H}(i)$ are i.i.d. random matrices taking values in the finite set $\mathcal{M}_{\zeta} \doteq\left\{M_{0}, M_{1}, \ldots, M_{\ell_{y}+\ell_{u}+2}\right\}$, where $M_{0}=I_{n_{\zeta}}$ and $M_{j}$ is defined as $M_{j} \doteq \operatorname{diag}\left\{M_{j}^{y}, M_{j}^{u}\right\}$, where

$$
\begin{aligned}
& M_{j}^{\star} \doteq {\left[\begin{array}{cc}
\Delta^{\star}(j) & 0 \\
I-\Delta^{\star}(j) & \Gamma^{\star}(j)
\end{array}\right], } \\
& \Delta^{\star}(j) \doteq \operatorname{diag}\left\{\delta_{1}^{\star}(j) I_{n_{y}}, \ldots, \delta_{\ell_{\star}}^{\star}(j) I_{n_{y}}\right\}, \\
& \Gamma^{\star}(j) \doteq\left[\begin{array}{cc}
\gamma_{1}^{\star}(j) I_{n_{y}} & \ddots \\
\left(1-\gamma_{1}^{\star}(j)\right) I_{n_{y}} \gamma_{2}^{\star}(j) I_{n_{y}} & \ddots \\
\ddots & \left(1-\gamma_{\ell_{\star}-1}^{\star}(j)\right) I_{n_{y}} \gamma_{\ell_{\star}}^{\star}(j) I_{n_{y}}
\end{array}\right],
\end{aligned}
$$

with $\star \in\{y, u\}$ and: $\delta_{\alpha}^{y}(j)=0$ if $j=\alpha$ and equal to 1 otherwise; $\delta_{\alpha}^{u}(j)=0$ if $j=\alpha+\ell_{y}+1$ and equal to 1 otherwise; $\gamma_{\alpha}^{y}(j)=0$ if $j=\alpha+1$ and equal to 1 otherwise; and $\gamma_{\alpha}^{u}(j)=$ 0 if $j=\alpha+\ell_{y}+2$ and equal to 1 otherwise, for $\alpha=1, \ldots, \ell_{y}$ and $\beta=1, \ldots, \ell_{u}$.

The set $\mathcal{M}_{\zeta}$ is the set containing all possible matrices that result from each contending device $D_{1}^{y}, \ldots, D_{\ell_{y}}^{y}, D_{1}^{u}, \ldots, D_{\ell_{u}}^{u}$ getting access to the timeslot. For instance, $M_{1}$ corresponds to the case where $D_{1}^{y}$ gets timeslot access to sample the plant measurement, $M_{2}$ corresponds to the case where $D_{1}^{y}$ gets timeslot access to transmit, and so on. More details regarding the construction of these matrices can be found in [5], [6].

Therefore, at a given transmission instant $t_{i}, \mathcal{H}(i)$ will be equal to some $M_{j}, j=0,1, \ldots, \ell_{y}+\ell_{u}+2$. In addition, each transmission can be subject to packet loss. We recall that transmissions are modelled by zeroing components of the network-induced error vector, so when packets are lost, the error remains unchanged, i.e. we admit the possibility of $\mathcal{H}(i)=M_{0}=I_{n_{\zeta}}$ with non-zero probability.

We adopt the following assumption on the stochastic protocol matrices $\{\mathcal{H}(i)\}_{i \in \mathbb{N}}$.

Assumption 2: $\mathcal{H}(i) \in \mathcal{M}_{\zeta}$ are i.i.d. random matrices with associated probabilities $\mathbf{P}\left\{\mathcal{H}(i)=M_{j}\right\}=p /\left(\ell_{y}+\ell_{u}+2\right)$ for all $i \in \mathbb{N}$ and $j \in\left\{1, \ldots, \ell_{y}+\ell_{u}+2\right\}$, where $p$ is the successful transmission probability.

Assumption 2 states that every field device is equally likely to access the timeslot and transmit successfully. The probability of having a packet dropout is $\mathbf{P}\left\{\mathcal{H}(i)=M_{0}\right\}=1-p$. Having any other distribution of probabilities may lead to some field devices being favoured over others during contention, which is not the case in WH.

Remark 1: Although the adopted modelling framework is presented for WH networks by including simplex device dynamics and multi-hop structure, our results can be applied 


$$
\begin{aligned}
f(x, \zeta, w) & \doteq\left(f_{p}\left(x_{p}, \mathbf{I}_{n_{u}}^{2 \ell_{u}} \cdot \zeta^{u}+g_{c}\left(x_{c}\right), w\right), f_{c}\left(x_{c}, \mathbf{I}_{n_{y}}^{2 \ell_{y}} \cdot \zeta^{y}+g_{p}\left(x_{p}\right), w\right)\right), \\
g(x, \zeta, w) & \doteq\left(-\frac{\partial g_{p}}{\partial x_{p}} f_{p}\left(x_{p}, \mathbf{I}_{n_{u}}^{2 \ell_{u}} \cdot \zeta^{u}+g_{c}\left(x_{c}\right), w\right), 0, \ldots, 0,-\frac{\partial g_{c}}{\partial x_{c}} f_{c}\left(x_{c}, \mathbf{I}_{n_{y}}^{2 \ell_{y}} \cdot \zeta^{y}+g_{p}\left(x_{p}\right), w\right), 0, \ldots, 0\right),
\end{aligned}
$$

to other wireless multi-hop networks with minor modifications. For example, we can cover multi-hop networks with duplex devices (i.e. they can receive and transmit at the same time) by simply redefining the set $M_{\zeta}$ accordingly.

\section{MAIN RESULTS}

In this section, we present a hybrid model for the WNCS in Fig. 2, and then we use it to obtain our stability result. Define the augmented state $x \doteq\left(x_{p}, x_{c}\right)$, where $x \in \mathbb{R}^{n_{x}}, n_{x} \doteq$ $n_{p}+n_{c}$. By using (2), (3), (4), and (5), we obtain the following model for the block diagram in Fig. 2,

$$
\begin{aligned}
\dot{x} & =f(x, \zeta, w), \quad t \in\left[t_{i}, t_{i+1}\right], \\
\dot{\zeta} & =g(x, \zeta, w), \quad t \in\left[t_{i}, t_{i+1}\right], \\
x\left(t_{i}^{+}\right) & =x\left(t_{i}\right), \\
\zeta\left(t_{i}^{+}\right) & =\mathcal{H}(i) \zeta\left(t_{i}\right),
\end{aligned}
$$

where $f$ and $g$ are given in (6), the transmission instants $\left\{t_{i}\right\}_{i \in \mathbb{N}}$ satisfy Assumption 1, and the stochastic matrices $\{\mathcal{H}(i)\}_{i \in \mathbb{N}}$ satisfy Assumption 2.

Please note that the network-induced error dynamics in (6b) have a particular structure in which two of its components are non-zero while the rest are zero. To simplify analysis, we re-arrange the error vector $\zeta$, i.e. we consider $\zeta \doteq \mathcal{T} \zeta$, where $\mathcal{T}$ is such that the first two components of $\dot{\zeta}$ are the non-zero components of $\dot{\zeta}$, and the rest are zero (see (6b)). The definition of $\mathcal{T}$ is straightforward and thus not included given space constraints. This mathematical structure is key in the following stability results, and it comes directly from the multi-hop nature of WH.

We now provide sufficient conditions for $\mathcal{L}_{p}$ stability-inexpectation of the stochastic WNCS in (7). We start with some important definitions.

Definition 2: Consider the protocol equation (7d) and suppose the stochastic matrices $\{\mathcal{H}(i)\}_{i \in \mathbb{N}}$ satisfy Assumption 2. Define $T_{0} \doteq \min \left\{j \geq 1:\left\{M_{1}, \ldots, M_{\ell_{y}+\ell_{u}+2}\right\} \subset\right.$ $\{\mathcal{H}(0), \ldots, \mathcal{H}(j-1)\}\}$, and inductively for $i>0$, $T_{i} \doteq \min \left\{j \geq 1:\left\{M_{1}, \ldots, M_{\ell_{y}+\ell_{u}+2}\right\} \subset\right.$ $\left.\left\{\mathcal{H}\left(T_{i-1}\right), \ldots, \mathcal{H}\left(T_{i-1}+j-1\right)\right\}\right\}$. We refer to $T_{i}$ as the $i$-th cover time, and collectively the cover time process.

Note that since $\{\mathcal{H}(i)\}_{i \in \mathbb{N}}$ is i.i.d., then $\left\{T_{i}\right\}_{i \in \mathbb{N}}$ is a stationary process.

Definition 3: Let $\tau_{i}=t_{i}-t_{i-1}$ satisfy Assumption 1 . We say that $C(j, k) \doteq\left\{\left(\mathcal{H}(j), \tau_{j}\right), \ldots,\left(\mathcal{H}(k), \tau_{k}\right)\right\}$ is a covering sequence if and only if $\left\{M_{1}, \ldots, M_{\ell_{y}+\ell_{u}+2}\right\} \subset$ $\{\mathcal{H}(j), \ldots, \mathcal{H}(k)\}$.

Note that cover times are simply the lengths of consecutive disjoint covering sequences.

Remark 2: Definitions 2 and 3 will help us define the stochastic analogue of the PE property previously introduced in [15]. PE was also used in the context of WH in [6] for
TDMA schemes. This property states that every field device is visited at least once after $T$ transmissions. Note that the value of $T$ is found from the implemented deterministic scheduling protocol. However, for stochastic schemes like CSMA-CA we need to study the probability distribution of $T_{i}$. We can find this distribution via solving the coupon collector's problem [17]. Let $n$ objects be picked repeatedly and equally likely, with replacement. The statement of interest is to find how many objects we expect to pick before having picked each object at least once. In multiple-access schemes, field devices are randomly getting timeslot access to transmit, and we thus need to find the expected number of transmissions $\mathbf{E}\left\{T_{i}\right\}$ in which all matrices $\left\{M_{1}, \ldots, M_{\ell_{y}+\ell_{u}+2}\right\}$ have been covered at least once.

The solution for the coupon collector's problem has a closed form expression [17], and applied to our case is given by $\mathbf{E}\{T\}=\left(\ell_{y}+\ell_{u}+2\right) H_{\ell_{y}+\ell_{u}+2} / p$, where $H_{n}$ is the $n$-th harmonic number, and we have dropped the time index $i$ in $T_{i}$ since $T_{i}$ is stationary. Intuitively, $T_{i}=\mathbf{E}\{T\}$ "most of the time" and $\mathbf{P}\left\{T_{i}<\infty\right\}=1$.

We are now ready to present the stochastic analogue of the PE property, which was firstly introduced in [12].

Definition 4: We say that a protocol is a.s. covering or has an a.s. finite cover time if in Definition 2, $\mathbf{P}\left\{T_{i}<\infty\right\}=1$ for all $i \in \mathbb{N}$.

Note that from the preceding discussion, this property is verified by the stochastic matrices $\{\mathcal{H}(i)\}_{i \in \mathbb{N}}$ that satisfy Assumption 2.

We make the following assumption, which was also used in [6] in for deterministic TDMA schemes.

Assumption 3: Let $L_{11} \in \mathcal{A}_{\geq 0}^{n_{\zeta_{1}^{y}}+n_{\zeta_{1}^{u}}}$ and $L_{12} \doteq$ $L_{11} \operatorname{diag}\left\{\mathbf{I}_{n_{y}}^{2 \ell_{y}-1}, \mathbf{I}_{n_{u}}^{2 \ell_{u}-1}\right\}$. There exists a matrix $A \in \mathcal{A}_{\geq 0}^{n_{\zeta}}$ of the form

$$
A=\left[\begin{array}{cc}
L_{11} & L_{12} \\
0 & 0
\end{array}\right]
$$

and a continuous function $\tilde{y}: \mathbb{R}^{n_{x}} \times \mathbb{R}^{n_{w}} \rightarrow \mathbb{R}_{\geq 0}^{n_{\zeta}}$ such that the error dynamics (7b) satisfy $\overline{\boldsymbol{g}}(x, \zeta, w) \preceq A \overline{\boldsymbol{\zeta}}+\tilde{y}(x, w)$, for all $(x, \boldsymbol{\zeta}, w) \in \mathbb{R}^{n_{x}} \times \mathbb{R}^{n_{\zeta}} \times \mathbb{R}^{n_{w}}$.

Assumption 3 is natural in WH networks given its multi-hop structure. More details can be found in [6].

The following theorem provides closed-loop $\mathcal{L}_{p}$ stabilityin-expectation for the WNCS (7).

Theorem 1: Consider the WNCS (7) and assume that

(i) The stochastic protocol is such that Assumption 2 is satisfied (i.e., it is an a.s. covering protocol), and Assumption 3 holds with $\tilde{y}=G(x)+w$.

(ii) The $x$-subsystem in (7) is $\mathcal{L}_{p}$ stable-in-expectation from $(\boldsymbol{\zeta}, w)$ to $G(x)$ with expected gain $\gamma$ for some $p \in[1, \infty]$. 
(iii) There exists $\boldsymbol{\omega}<\infty$ such that $\gamma_{\boldsymbol{\omega}} \gamma<1, \boldsymbol{\omega}>\left|L_{11}\right| / p$ and $\rho_{\boldsymbol{\omega}}<1$ hold, where $\gamma_{\boldsymbol{\omega}} \doteq \mathbf{E}\{T\}\left(1+\rho_{\boldsymbol{\omega}}\right) /((\boldsymbol{\omega}-$ $\left.|A|)\left(1-\rho_{\boldsymbol{\omega}}\right)\right)$, and

$$
\begin{aligned}
& \rho_{\boldsymbol{\omega}} \doteq\left(\left(\alpha_{\boldsymbol{\omega}} p\right)^{V}\right. \\
& \left.\prod_{j=1}^{V} \frac{V-(j-1)}{V\left(1-(1-p) \alpha_{\boldsymbol{\omega}}\right)-(j-1) p \alpha_{\boldsymbol{\omega}}}-1\right) \sqrt{\ell},
\end{aligned}
$$

with $V \doteq \ell_{y}+\ell_{u}+2, \alpha_{\boldsymbol{\omega}} \doteq \boldsymbol{\omega} /\left(\boldsymbol{\omega}-\left|L_{11}\right|\right)$, and $\ell \doteq$ $\max \left\{2 \ell_{y}, 2 \ell_{u}\right\}$.

Then, the WNCS (7) is $\mathcal{L}_{p}$ stable-in-expectation from $w$ to $(G(x), \zeta)$ with a finite linear expected gain.

Theorem 1 asserts that a.s. covering protocols lead to stability of the WNCS whenever transmissions occur fast enough. Particularly, it utilises small-gain arguments (related to conditions (i) and (ii)) to provide a choice of intensity of transmission $\omega$ that ensures stability of the WNCS. In fact, this choice can be found by numerically solving for $\boldsymbol{\omega}$ in (iii) under its corresponding constraints.

In comparison to [12], our results are tailored to multi-hop networks with CSMA-CA schemes as the intensity $\boldsymbol{\omega}$ depends on the number of field devices in each path $\ell_{y}, \ell_{u}$, and also the norm $\left|L_{11}\right|$ which comes directly from our model structure (see Assumption 3).

\section{NumericAl EXAMPLE}

Consider a non-linear plant $\dot{x}_{1}=-2 x_{1}-x_{1}^{3}+x_{2}, \dot{x}_{2}=$ $x_{2}^{2}-x_{2}^{3}+u$ with output $y=x_{2}$, a controller $u=-2 \hat{y}$, and a WH network with $\ell_{y}=2$ and $\ell_{u}=0$. We compute below the required intensity $\boldsymbol{\omega}$ for closed-loop $\mathcal{L}_{p}$ stabilityin-expectation. With this, we can also compute the expected transmission rate bounds as a function of the probability $p$. For this example, we can show that

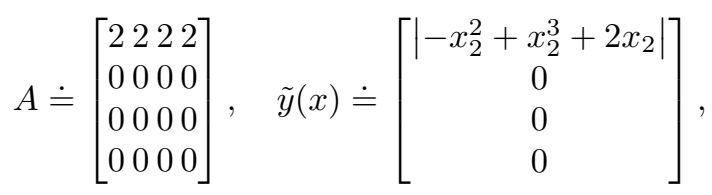

and we note that $L_{11}=2,|A|=4$, and $\left|L_{11}\right|=2$. We can compute the $\mathcal{L}_{2}$ gain from $\zeta$ to $\tilde{y}=\overline{A_{21} x}$ proceeding similarly to [18, Example 1] and get $\gamma=6.172$. With the above, we can numerically solve for $\boldsymbol{\omega}$ in Theorem 1 to find the intensity bound $\boldsymbol{\omega}_{\mathrm{thm} .1}^{*}$ that ensures closed-loop $\mathcal{L}_{p}$ stability-inexpectation for different values of probability $p$.

TABLE I

INTENSITY OF TRANSMISSION AND INTER-TRANSMISSION TIME BOUNDS WITH CSMA/CA AND PACKET DROPOUTS.

\begin{tabular}{||c||c|c||c|c||}
\hline$p$ & $\boldsymbol{\omega}_{\text {thm. }}^{*}$ & $\mathbf{E}\left\{\tau_{\text {thm. } 1}^{*}\right\}[\mathrm{ms}]$ & $\boldsymbol{\omega}_{[12]}^{*}$ & $\mathbf{E}\left\{\tau_{[12]}^{*}\right\}[\mathrm{ms}]$ \\
\hline 0.2 & 534.9 & 1.9 & 560.7 & 1.8 \\
\hline 0.4 & 268.5 & 3.7 & 281.3 & 3.6 \\
\hline 0.6 & 179.7 & 5.6 & 188.2 & 5.3 \\
\hline 0.8 & 135.3 & 7.4 & 141.7 & 7.1 \\
\hline 1 & 108.7 & 9.2 & 113.7 & 8.8 \\
\hline
\end{tabular}

Recall that the inter-transmission time is exponentially distributed, and it describes the time between events in a Poisson point process of intensity $\boldsymbol{\omega}$. Thus, we compute the expected inter-transmission time bound as $\mathbf{E}\left\{\tau^{*}\right\}=1 / \boldsymbol{\omega}^{*}$, for

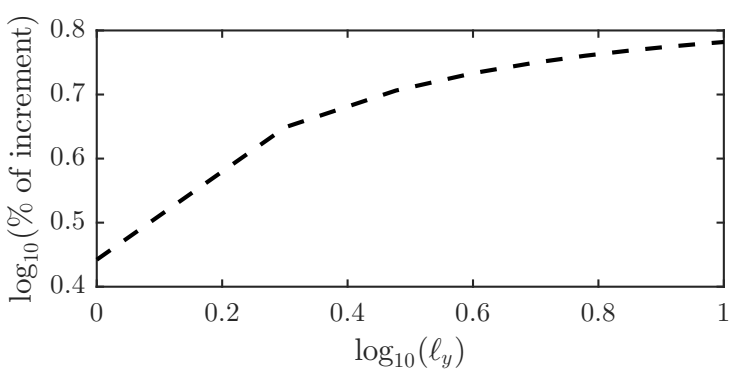

Fig. 3. Percentage of improvement between $\boldsymbol{\omega}_{\mathrm{thm} .1}^{*}$ and $\boldsymbol{\omega}_{[12]}^{*}$, for $p=0.8$ as the number of field devices in the $y$-path increases as per $\ell_{y} \in[1,10]$.

a given intensity bound $\boldsymbol{\omega}^{*}$. We also compute $\boldsymbol{\omega}_{[12]}^{*}$, obtained by using the results from [12], which do not exploit the potential structure of $A$. The above is summarised in Table I. It can be seen that our intensity bounds are smaller (better) than the previous bounds in [12], particularly we provide a percentage of improvement around 5\% for this example. More importantly, this improvement increases with the number of field devices as it can be seen in Fig. 3 .

From Table I, we see that the intensity of transmission grows as the successful transmission probability $p$ gets smaller. That is, the more defective the channel is, packets must be transmitted at a faster rate in order to preserve the $\mathcal{L}_{p}$ stabilityin-expectation of the WNCS. That is, the closer we are to an ideal channel $(p=1)$, the less intensity we require, which is in accordance with intuition.

\section{CONCLUSIONS}

We presented an analysis framework for non-linear systems closed over a WH network subject to packet dropouts and CSMA-CA schemes. Specifically, we presented a hybrid NCS model which we used to provide a bound on the intensity of transmission that ensures robust stability. Future work includes higher-fidelity modelling of the random back-off periods that field devices encounter when contesting a timeslot in CSMACA.

\section{APPENDIX}

\section{A. Proof of Theorem 1}

The following results are needed to prove Theorem 1 . Throughout this proof we define $Q_{i} \doteq \mathcal{H}(i)$.

Lemma 1: Let $T$ be the cover time for the sequence $\left\{\left(Q_{0}, \tau_{0}\right), \ldots,\left(Q_{T-1} \tau_{T-1}\right)\right\}$. Given Assumption 3, then the following inequality holds.

$$
\left|\left(\prod_{i=0}^{T-1} Q_{i} \exp \left(A \tau_{i}\right)\right)\right| \leq\left(\exp \left(\left|L_{11}\right| \sum_{i=0}^{T-1} \tau_{i}\right)-1\right) \sqrt{\ell},
$$

where we recall that $\ell \doteq \max \left\{2 \ell_{y}, 2 \ell_{u}\right\}$.

Proof: By a straightforward generalisation of Lemma 7.1 in [15], we have that

$$
\left(\prod_{i=0}^{T-1} Q_{i} \exp \left(A \tau_{i}\right)\right) \preceq\left(\prod_{i=0}^{T-1} Q_{i}\right)+\exp \left(A \sum_{i=0}^{T-1} \tau_{i}\right)-I .
$$


Then, given that $\left\{\left(Q_{0}, \tau_{0}\right), \ldots,\left(Q_{T-1} \tau_{T-1}\right)\right\}$ is a covering sequence, we have that

$$
\begin{aligned}
\left|\left(\prod_{i=0}^{T-1} Q_{i} \exp \left(A \tau_{i}\right)\right)\right| & \leq\left|\prod_{i=0}^{T-1} Q_{i}\right|+\left|\exp \left(A \sum_{i=0}^{T-1} \tau_{i}\right)-I\right| \\
& =\left|\exp \left(A \sum_{i=0}^{T-1} \tau_{i}\right)-I\right| .
\end{aligned}
$$

The proof follows by following the same steps after (A.1) in the proof of Lemma 14 in [6].

Remark 3: Let $W_{j}$ denote the number if additional transmissions needed to go from having covered $j-1$ matrices to $j$ matrices in $\mathcal{M}_{\zeta}$. Clearly $W_{j}$ is geometrically distributed with parameter $p_{g, j}$ given by $p_{g, j}=(V-j+1) p / V$, where $V \doteq \ell_{y}+\ell_{u}+2$. It is clear that $T$, the time to cover all matrices $M_{1}, \ldots, M_{V}$, can be expressed as $T=\sum_{j=1}^{V} W_{j}$ and its pgf is thus given by [17]

$$
\begin{aligned}
\mathbf{G}_{T}(s) & \doteq \mathbf{E}\left\{s^{T}\right\}=\mathbf{E}\left\{s^{\left(W_{1}+\cdots+W_{V}\right)}\right\}=\mathbf{E}\left\{s^{W_{1}} \cdots s^{W_{V}}\right\} \\
& =(s p)^{V} \prod_{j=1}^{V} \frac{V-(j-1)}{V(1-(1-p) s)-(j-1) p s}
\end{aligned}
$$

for $|s|<1 /(1-p)$.

Lemma 2: Suppose that $\tau_{i} \sim \operatorname{Exp}(\boldsymbol{\omega})$. Let $T$ be the cover time for the sequence $\left\{\left(Q_{0}, \tau_{0}\right), \ldots,\left(Q_{T-1} \tau_{T-1}\right)\right\}$, and let $Z \doteq \exp \left(\left|L_{11}\right| \sum_{j=0}^{T-1} \tau_{i}\right)$. Then, $\mathbf{E}\{Z\}$ is given by

$$
\mathbf{E}\{Z\}=\left(\alpha_{\boldsymbol{\omega}} p\right)^{V} \prod_{j=1}^{V} \frac{V-(j-1)}{V\left(1-(1-p) \alpha_{\boldsymbol{\omega}}\right)-(j-1) p \alpha_{\boldsymbol{\omega}}},
$$

where $\alpha_{\boldsymbol{\omega}} \doteq \boldsymbol{\omega} /\left(\boldsymbol{\omega}-\left|L_{11}\right|\right)$ and $\boldsymbol{\omega}>\left|L_{11}\right| / p$.

Proof: Let $W=\sum_{i=0}^{T-1} \tau_{i}$. Via Example 1.8.13 in [19], we have that the mgf of $W$ is given by $\mathbf{E}\{\exp (s W)\}=$ $\mathbf{G}_{T}\left(\mathbf{M}_{\tau}(s)\right)=\mathbf{G}_{T}(\boldsymbol{\omega} /(\boldsymbol{\omega}-s))$, where $\mathbf{M}_{\tau}$ is the mgf of the exponentially distributed random variables $\tau_{i} \sim \operatorname{Exp}(\boldsymbol{\omega})$, and $\mathbf{G}_{T}$ is given in (8). Therefore, $\mathbf{E}\{Z\}=\mathbf{E}\left\{\exp \left(\left|L_{11}\right| W\right)\right\}=$ $\mathbf{G}_{T}\left(\boldsymbol{\omega} /\left(\boldsymbol{\omega}-\left|L_{11}\right|\right)\right)$ for $\boldsymbol{\omega} /\left(\boldsymbol{\omega}-\left|L_{11}\right|\right)<1 /(1-p)$, completing the proof.

The following theorem asserts $\mathcal{L}_{p}$ stability-in-expectation for the $\zeta$-subsystem and is the key component of the smallgain-based proof approach behind Theorem 1 .

Theorem 2: Consider the WNCS (7) and assume that we employ a stochastic protocol that satisfies hypothesis (i) of Theorem 1. If there exists $\boldsymbol{\omega}<\infty$ such that $\rho_{\boldsymbol{\omega}}<1$, where $\rho_{\boldsymbol{\omega}} \doteq(\mathbf{E}\{Z\}-1) \sqrt{\ell}, \mathbf{E}\{Z\}$ as per Lemma 2 and $\ell \doteq \max \left\{2 \ell_{y}, 2 \ell_{u}\right\}$, then the $\zeta$-subsystem is $\mathcal{L}_{p}$ stable-inexpectation from $\tilde{y}$ to $\zeta$ with expected gain $\gamma_{\boldsymbol{\omega}} \doteq \mathbf{E}\{T\}(1+$ $\left.\rho_{\boldsymbol{\omega}}\right) /\left((\boldsymbol{\omega}-|A|)\left(1-\rho_{\boldsymbol{\omega}}\right)\right)$.

Proof: By choosing $\boldsymbol{\omega}$ such that $\rho_{\boldsymbol{\omega}}<1$, we have that via Lemma 1, $\mathbf{E}\left\{\left|\left(\prod_{i=0}^{T-1} Q_{i} \exp \left(A \tau_{i}\right)\right)\right|\right\}<1$. With this, the remainder of the proof follows by mimicking the proof steps of Theorem 9.4 in [12] with $\rho=\rho_{\boldsymbol{\omega}}$ and it is thus omitted.

We are now in a position to prove Theorem 1 . Consider the small-gain theorem for impulsive systems in [14, Theorem 1]. This theorem can be easily extended to the stochastic case by the use of Definition 1. Given hypothesis (i) in Theorem 1 and the fact that $\boldsymbol{\omega}$ is chosen such that $\rho_{\boldsymbol{\omega}}<1$, then we have
$\mathcal{L}_{p}$ stability-in-expectation of the $\boldsymbol{\zeta}$-subsystem via Theorem 2 , from $(G(x), w)$ to $\zeta$ with gain $\gamma_{\boldsymbol{\omega}}$. Because assertion (ii) of Theorem 1 states $\mathcal{L}_{p}$ stability-in-expectation of the $x-$ subsystem from $(\boldsymbol{\zeta}, w)$ to $G(x)$ with gain $\gamma$, we can resort to the aforementioned stochastic small-gain theorem, which concludes the proof of Theorem 1 .

\section{REFERENCES}

[1] P. Park, S. C. Ergen, C. Fischione, C. Lu, and K. H. Johansson, "Wireless network design for control systems: A survey," IEEE Communications Surveys \& Tutorials, vol. 20, no. 2, pp. 978-1013, 2018.

[2] "HART Communication Protocol," https://www.fieldcommgroup.org/ technologies/hart, FieldComm Group.

[3] R. Alur, A. d'Innocenzo, K. Johansson, G. Pappas, and G. Weiss, "Compositional modeling and analysis of multi-hop control networks," IEEE Transactions on Automatic control, vol. 56, no. 10, pp. 2345-2357, 2011.

[4] B. Demirel, Z. Zou, P. Soldati, and M. Johansson, "Modular design of jointly optimal controllers and forwarding policies for wireless control," IEEE Transactions on Automatic Control, vol. 59, no. 12, pp. 3252$3265,2014$.

[5] A. Maass, D. Nešić, R. Postoyan, P. Dower, and V. Varma, "Emulationbased stabilisation of networked control systems over WirelessHART," in Proceedings of the 56th IEEE Conference on Decision and Control, Melbourne, Australia, 2017, pp. 6628-6633.

[6] A. Maass, D. Nešić, R. Postoyan, and P. Dower, " $\mathcal{L}_{p}$ stability of networked control systems implemented on WirelessHART," Provisionally accepted in Automatica, 2019.

[7] B. Demirel, A. Aytekin, D. E. Quevedo, and M. Johansson, "To wait or to drop: On the optimal number of retransmissions in wireless control," in Control Conference (ECC), 2015 European. IEEE, 2015, pp. 962 968.

[8] P. Park, P. Di Marco, P. Soldati, C. Fischione, and K. Johansson, "A generalized markov chain model for effective analysis of slotted IEEE 802.15.4," in 2009 IEEE 6th International Conference on Mobile Adhoc and Sensor Systems, 2009, pp. 130-139.

[9] P. Park, P. Di Marco, C. Fischione, and K. Johansson, "Modeling and optimization of the IEEE 802.15.4 protocol for reliable and timely communications," IEEE Transactions on Parallel and Distributed Systems, vol. 24, no. 3, pp. 550-564, 2013.

[10] P. Park, J. Araújo, and K. Johansson, "Wireless networked control system co-design," in 2011 International Conference on Networking, Sensing and Control. IEEE, 2011, pp. 486-491.

[11] L. Zou, Z. Wang, and H. Gao, "Observer-based $H_{\infty}$ control of networked systems with stochastic communication protocol: The finitehorizon case," Automatica, vol. 63, pp. 366-373, 2016.

[12] M. Tabbara and D. Nešić, "Input-output stability of networked control systems with stochastic protocols and channels," IEEE Transactions on Automatic control, vol. 53, no. 5, pp. 1160-1175, 2008.

[13] G. Walsh, O. Beldiman, and L. Bushnell, "Asymptotic behavior of nonlinear networked control systems," IEEE Transactions on Automatic Control, vol. 46, no. 7, pp. 1093-1097, jul 2001.

[14] D. Nešić and A. Teel, "Input-output stability properties of networked control systems," IEEE Transactions on Automatic Control, vol. 49, no. 10, pp. 1650-1667, 2004.

[15] M. Tabbara, D. Nešić, and A. Teel, "Stability of wireless and wireline networked control systems," IEEE Transactions on Automatic Control, vol. 52, no. 9, pp. 1615-1630, 2007.

[16] U. Feige, "A tight upper bound on the cover time for random walks on graphs," Random Structures \& Algorithms, vol. 6, no. 1, pp. 51-54, 1995.

[17] J. Pitman, "Probability," 1993.

[18] D. Nešić, A. R. Teel, and D. Carnevale, "Explicit computation of the sampling period in emulation of controllers for nonlinear sampled-data systems," IEEE Transactions on Automatic Control, vol. 54, no. 3, pp. 619-624, 2009.

[19] D. Stirzaker et al., "Stochastic processes and models," OUP Catalogue, 2005. 